This is the post peer-review accepted manuscript of:

V. Jelicic et al., "Towards Internet of Things for event-driven low-power gas sensing using carbon nanotubes," 2015 6th International Workshop on Advances in Sensors and Interfaces (IWASI), Gallipoli, 2015, pp. 271-276.

The published version is available online at:

https://doi.org/10.1109/IWASI.2015.7184980

(C)2015 IEEE. Personal use of this material is permitted. Permission from IEEE must be obtained for all other uses, in any current or future media, including reprinting/republishing this material for advertising or promotional purposes, creating new collective works, for resale or redistribution to servers or lists, or reuse of any copyrighted component of this work in other works. 


\title{
Towards Internet of Things for Event-Driven Low-Power Gas Sensing using Carbon Nanotubes
}

\author{
Vana Jelicic*, Michele Magno ${ }^{\dagger}$, Kiran Chikkadi ${ }^{\ddagger}$, Cosmin Roman $^{\ddagger}$, Christofer Hierold ${ }^{\ddagger}$, Vedran Bilas*, and \\ Luca Benini ${ }^{\dagger}$ \\ *University of Zagreb, Faculty of Electrical Engineering and Computing \\ Zagreb, Croatia \\ e-mail: vana.jelicic@fer.hr \\ $\dagger$ Dept. of Information Technology and Electrical Engineering, ETH Zurich \\ Zurich, Switzerland \\ e-mail: michele.magno@iis.ee.ethz.ch \\ ${ }_{\ddagger}^{\ddagger}$ Department of Mechanical and Process Engineering, ETH Zurich \\ Zurich, Switzerland
}

\begin{abstract}
One of most important applications of sensing devices under the Internet of Things paradigm is air quality monitoring, which is particularly useful in urban and industrial environments where air pollution is an increasing public health problem. As these sensing systems are usually battery-powered and gas sensors are power-hungry, energy-efficient design and power management are required to extend the device's lifetime. In this paper, we present a two-stage concept where a novel lowpower carbon nanotube is used as a gas detector for an energyconsuming metal-oxide (MOX) semiconductor gas sensor. We propose a design of a heterogeneous sensor node where we exploit the low-power nanotube gas sensor and the more accurate MOX sensor. This work performs energy consumption simulations for three event-driven scenarios to evaluate the power consumption reduction, as well as the limitations of carbon nanotubes. Our results show the benefits of the proposed approach over the scenarios with adaptive duty-cycling with only MOX gas sensors, proved with $20 \%-35 \%$ node lifetime prolongation. The delay introduced due to the nanotube recovery time can be overcome by radio duty-cycled activity for detecting alarm messages from the neighbour nodes.
\end{abstract}

\section{INTRODUCTION}

Recent technology advances have led to the development of embedded devices encompassing sensing, computing and communication, enabling the concept of Internet of Things (IoT) where the devices are connected to the Internet. These ubiquitous platforms, also known as wireless sensor devices have been recognized as a fundamental enabling technology for a large variety of applications including environmental monitoring, healthcare, security [1-3], industrial monitoring [4], etc. Air quality monitoring is becoming a popular application as pollution is an increasing problem in urban areas, directly connected to the density increment of combustion-powered vehicles. Pollutants such as carbon monoxide (CO), nitrogen dioxide $\left(\mathrm{NO}_{2}\right)$, and sulphur dioxide $\left(\mathrm{SO}_{2}\right)$ are harmful to human health when found above certain concentrations in the air. Moreover, in indoor ambient higher concentrations of $\mathrm{CO}, \mathrm{CH}_{4}$ and other gases can cause discomfort, severe health problems or even death. That is why health-based air quality standards and objectives are widely used in many countries $[5,6]$.
Air quality in cities is measured by monitoring stations; usually only a few of them distributed within the city area. As the pollutant concentration varies greatly according to the distance from the traffic, as well as to the topography and weather conditions, it would be useful to monitor personal exposure to harmful gases. There are several mobile sensing projects supporting the IoT paradigm, where citizens or public transport vehicles are equipped with sensing devices which measure gas concentration and send the information to the Internet [7-9]. Such systems of mobile gas sensing devices ensure a larger spatial and temporal density of gas concentration information, giving a better coverage of city area and providing information for near-real-time pollution maps. On the other hand, in indoor areas, wireless networks of densely deployed gas sensors can be used for monitoring air quality and for ensuring a prompt reaction in case of a hazardous event such as gas leakage [10]. As the wireless embedded sensing devices usually are batterypowered, their limited energy budget presents a research and design challenge. There are different gas sensor technologies that can be used in such devices [11].

Metal-Oxide Semiconductor (MOX) gas sensors are cheap, small in size and lightweight, which is beneficial for their use in pervasive mobile sensing systems for environmental monitoring [12]. Their biggest downsides are problems related to drift and especially their significant energy consumption. This energy is needed to activate the interaction with a certain gas for concentration measurements, and the sensing layer of a MOX gas sensor needs to be heated up for long periods (few minutes). Manufacturer instructions usually propose continuous heating with up to $100 \mathrm{~mW}$ power, which is too high for small-size batteries. Thus, in order to prolong the battery lifetime of gas sensor nodes-which also has to communicate the data wirelessly-research has been done to save energy of MOX gas sensors, with power management reducing the activity of the gas sensors.

An alternative, emerging technology for ultra-low-power gas sensors is based on carbon nanotubes. In recent years, carbon nanotubes have been explored for gas detection at ambient temperature, while self-heating was shown to accelerate gas desorption. However, this technology is still in its infancy and issues such as long response and recovery times as well as 
unknown long-term stability currently hinder its use as a direct replacement for MOX sensors [13]. More investigations into their drift, stability and response times are thus required before using them as stand-alone gas concentration monitors. On the other hand, nanotube technology shows a good potential for sensor network applications, air quality monitoring and breath analysis systems using battery-operated devices, as they have a very low power consumption (around few $\mu \mathrm{W}$ ) in continuous monitoring $[14,15]$.

In this paper, we introduce the concept of using a carbon nanotube gas sensor as an ultra-low-power wake-up detector for power-hungry MOX sensors. The proposed approach combines the benefits of both technologies to achieve ultra-lowpower systems ready for long lifetime IoT. The system exploits the benefits of low power consumption of the nanotube with the established accuracy of MOX sensors. With an aggressive power management it is possible to keep the energy-consuming MOX gas sensor inactive most of the time (i.e., with the heater turned off), while the pollutant concentration is continuously monitored by an ultra-low-power gas sensor.

The remainder of the paper is organized as follows. Section II provides an overview of the related work. Section III presents the low-power two-tier sensor wake-up method for gas concentration detection using a MOX gas sensor and a carbon nanotube gas detector. In Section IV, the architecture of the proposed gas sensor node is described. Section V and Section VI present the simulation setup and results, respectively. Conclusions and future work challenges are brought in Section VII.

\section{RELATED WORKS}

Reducing energy consumption of power-hungry gas sensors (in particular MOX sensors) has been a topic of intensive research in recent years. The most common energy-saving method for gas sensors is duty-cycling [16]. Oletic et al. [17] explore the minimal number of heating pulses necessary to establish MOX sensing layer's steady state. In $[10,18]$, the benefits of a heterogeneous gas sensing system with adaptive duty-cycling have been explored and improvements have been demonstrated in terms of lifetime extensions. Moreover, to further reduce a gas sensor's energy consumption, methods to recognize gas concentration from its transient response have been studied. In that way, the sensor does not need to be heated until it reaches steady state (which might take minutes), but less than a second [19, 20]. In [21], a two-stage concept for reducing energy consumption of MOX gas sensors by early detection of clean air from the transient sensor response has been proposed. Duty-cycling schemes have a huge advantage in terms of energy savings, but they come at the price of reducing the system's reactivity and high possibility of missing an important critical situation.

It was shown that some carbon nanotubes can detect $\mathrm{NO}_{2}$ gas in sub-ppm range-typical for atmosphere concentrations [6] - with less than $50 \mathrm{nW}$ power consumption in active state [14]. Gas sensors based on suspended carbon nanotubes can be recovered after $\mathrm{NO}_{2}$ exposure via self-heating. Full recovery can be achieved in less than 10 minutes and it requires the ultra-low power of $2.9 \mu \mathrm{W}$ [14]. In addition, crosssensitivity of nanotube sensors to gases other than $\mathrm{NO}_{2}$ is an important challenge, as it would generate false triggers. Surface functionalization of carbon nanotubes can be utilized to render them sensitive to other gases, such as $\mathrm{CO}$ [22] and $\mathrm{SO}_{2}$. Multiple analyte discrimination is also possible using nanotube sensor arrays [23]. Suspended, ultra-clean carbon nanotube gas sensors are also insensitive to humidity variations [24].

Carbon nanotube gas sensors are still in an early development phase to be used as stand-alone low-power gas concentration monitors, while commercially available MOX sensors are an established yet energy-consuming technology. In this work we seek to combine the advantages of the two technologies, by utilizing carbon nanotubes as ultra-low-power detectors of gas concentration changes to trigger MOX gas sensors for subsequent gas concentration measurements. This architecture will be shown to result in significant improvements in the energy efficiency of the gas sensor node and reactivity of the proposed solution. To the best of authors' knowledge, this is the first proposal of a nanotube gas sensor used as an eventdriven detector for MOX gas sensor networks. In addition, it is the first study of practical benefits of carbon nanotubes for energy-efficient pollution monitoring.

\section{CARBON NANOTUBE AS MOX GAS SENSOR TRIGGER}

We propose a two-tier sensor wake-up method that aims at reducing the activity of a MOX gas sensor as much as possible and increase the responsiveness of the system if gas concentration increase is detected. The proposed approach is presented in Fig. 1. Due to the presence of the nanotube gas sensor, the node can be in idle state most of the time. In this state, almost all of the node's components are switched off, including the MOX sensor and its heater. To ensure the reaction to a possible gas concentration increment, the carbon nanotube is continuously monitoring the gas concentration. When the gas concentration rises, it is detected by the carbon nanotube, and this alarm causes the MOX heater to turn on. The MOX sensor is heated until steady state of the sensing layer is reached and the gas concentration can be determined. At the same time, after alarm generation, the carbon nanotube has to be exposed to a high current for 10 minutes, to recover its response to a baseline and be ready for further sensing. In addition, it is possible to activate the MOX heater upon receiving an alarm message from a neighbour sensor node. To enable such functionality, the radio module has to periodically check for messages (i.e., work in a duty-cycled mode). The node states and activities of particular node components in each state are listed in Table I.

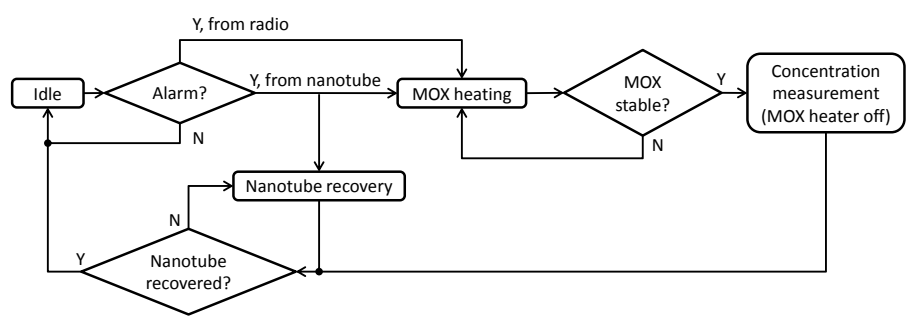

Fig. 1. State diagram showing the novel concept for low-power two-tier gas sensing where a MOX gas sensor is triggered by a carbon nanotube.

In order to estimate the benefits of this concept in terms of energy-efficient gas monitoring, we propose a sensor node 
TABLE I. NODE STATES AND ACTIVITY OF ITS COMPONENTS IN EACH STATE.

\begin{tabular}{|l||l|l|l|l|}
\hline State & Nanotube & Radio & $\begin{array}{l}\text { MOX } \\
\text { sensor heater }\end{array}$ & MCU \\
\hline \hline Idle & on, idle & $\begin{array}{l}\text { off } \\
\text { (duty cycle) }\end{array}$ & off & sleep \\
\hline MOX heating & recover & off & on & on \\
\hline $\begin{array}{l}\text { Concentration } \\
\text { measurement }\end{array}$ & recover & off & off & on \\
\hline $\begin{array}{l}\text { Nanotube } \\
\text { recovery }\end{array}$ & recover & $\begin{array}{l}\text { off } \\
\text { (duty cycle) }\end{array}$ & $\begin{array}{l}\text { off } \\
\text { (on, if alarm received) }\end{array}$ & sleep \\
\hline
\end{tabular}

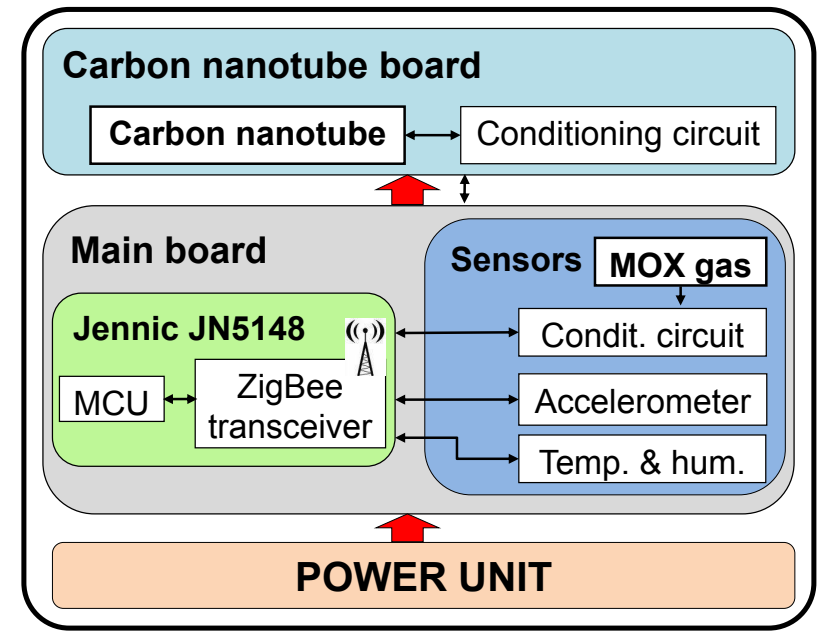

Fig. 2. Block scheme of the wireless gas sensor node.

design and perform a set of simulations exploring the energy consumption of the node.

\section{SENSOR NODE}

The block diagram of the proposed sensor node is shown in Fig. 2. The main board of the sensor node contains a microcontroller and a wireless communication module, as well as the sensors (including the MOX gas sensor). The carbon nanotube board would be connected over GPIO pins to provide the information about the gas concentration increment. The power unit contains two AA batteries. Table II comprises the values of power consumption for the node in different operating states.

TABLE II. SENSOR NODE POWER CONSUMPTION

(a) Main board

\begin{tabular}{|c|c|c||c|}
\hline \multicolumn{3}{|c||}{ State } & $\begin{array}{c}\text { Power consumption } \\
{[\mathrm{mW}]}\end{array}$ \\
\hline MCU & radio & MOX gas sensor & $24 \cdot 10^{-3}$ \\
\hline sleep & off & off & 50 \\
\hline on & off & on & 48 \\
\hline on & on, idle & off & 54 \\
\hline on & on, RX & off & 45 \\
\hline on & on, TX & off & \\
\hline
\end{tabular}

(b) Carbon nanotube board.

\begin{tabular}{|c|c|}
\hline State & Power consumption $[\mu \mathrm{W}]$ \\
\hline \hline on, idle & 20 \\
\hline gas detection & 20 \\
\hline recovery & 22.9 \\
\hline
\end{tabular}

\section{A. Main board}

The core of the sensor node is Wispes W24TH node [25]. It is based on Jennic JN5148 module, which is an ultra-lowpower, high performance microcontroller with ZigBee PRO networking capabilities. The power consumption of the module is $15 \mathrm{~mA}$ when transmitting and $18 \mathrm{~mA}$ when receiving a message. The board has several sensors (an accelerometer, a temperature and humidity sensor and a gas sensor with its conditioning circuit) useful for various ambient monitoring applications. A microSD card reader, intended for local backup, data logging and firmware update, is also present on the node. The power consumption of the main board in sleep state is very low $(24 \mu \mathrm{W})$.

1) MOX gas sensor: One of the commercially available $\mathrm{NO}_{2}$ sensors is MiCS-2710 from e2v technologies [26]. The sensor is very compact and covers a wide detection range. The recommended heater power is $43 \mathrm{~mW}$, in a constant power mode, which causes the temperature of the sensing layer to reach about $220^{\circ} \mathrm{C}$. The gas sensor is surrounded by a flexible conditioning circuit that enables the microcontroller to control the heater voltage and monitor the sensor resistance and current consumption. By modifying the heater voltage, it is possible to control the temperature of the sensing layer, which is responsible for the chemical reaction progress. Experiments show that the sensor response stabilizes after a certain time (usually several seconds, depending on the duration of inactive time that the sensor was in before turning on the heater) $[10,21]$. Thus, in order to save energy, we will turn off the heater when the sensor response reaches the steady state (approximately after 2 seconds).

\section{B. Carbon nanotube board}

The carbon nanotube [14] consumes less than $1 \mu \mathrm{W}$ when in ambient without $\mathrm{NO}_{2}$ pollution. When exposed to an increased $\mathrm{NO}_{2}$ concentration, its output changes. The response time is 90 minutes according to [14]. However, in our system where we use it as a trigger, we do not wait for the sensor output to reach saturation and we trigger the MOX sensor when the output reaches a critical value.

Fig. 3 shows the sensor response of a typical suspended carbon nanotube gas sensor. The top plot in Fig. 3 shows the sensor response calculated as the relative change in current $\left(\Delta \mathrm{I} / \mathrm{I}_{0}\right)$ upon exposure to a $1 \mathrm{ppm}$ concentration of $\mathrm{NO}_{2}$. The transfer characteristics of the carbon nanotube transistor, showing the change as a function of gate voltage is shown in the bottom plot. The bias voltage is $500 \mathrm{mV}$, so the power consumption in the idle state is about $600 \mathrm{nW}$. The undisturbed recovery time is unacceptably long, and has been reported

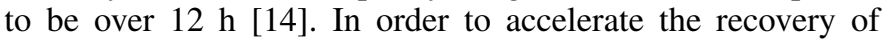
carbon nanotube sensors, self-heating is induced by increasing the current through the sensor. Fig. 4 shows the response of another device to $\mathrm{NO}_{2}$ exposure and self-heated recovery phases [14]. This device consumes $40 \mathrm{nW}$ during its idle phase, $80 \mathrm{nW}$ when exposed to $900 \mathrm{ppb} \mathrm{NO}_{2}$, and $2.9 \mu \mathrm{W}$ during the recovery phase.

The carbon nanotube requires conditioning circuitry (voltage converter providing appropriate voltage), and a comparator that generates a trigger to the microcontroller to turn on the 

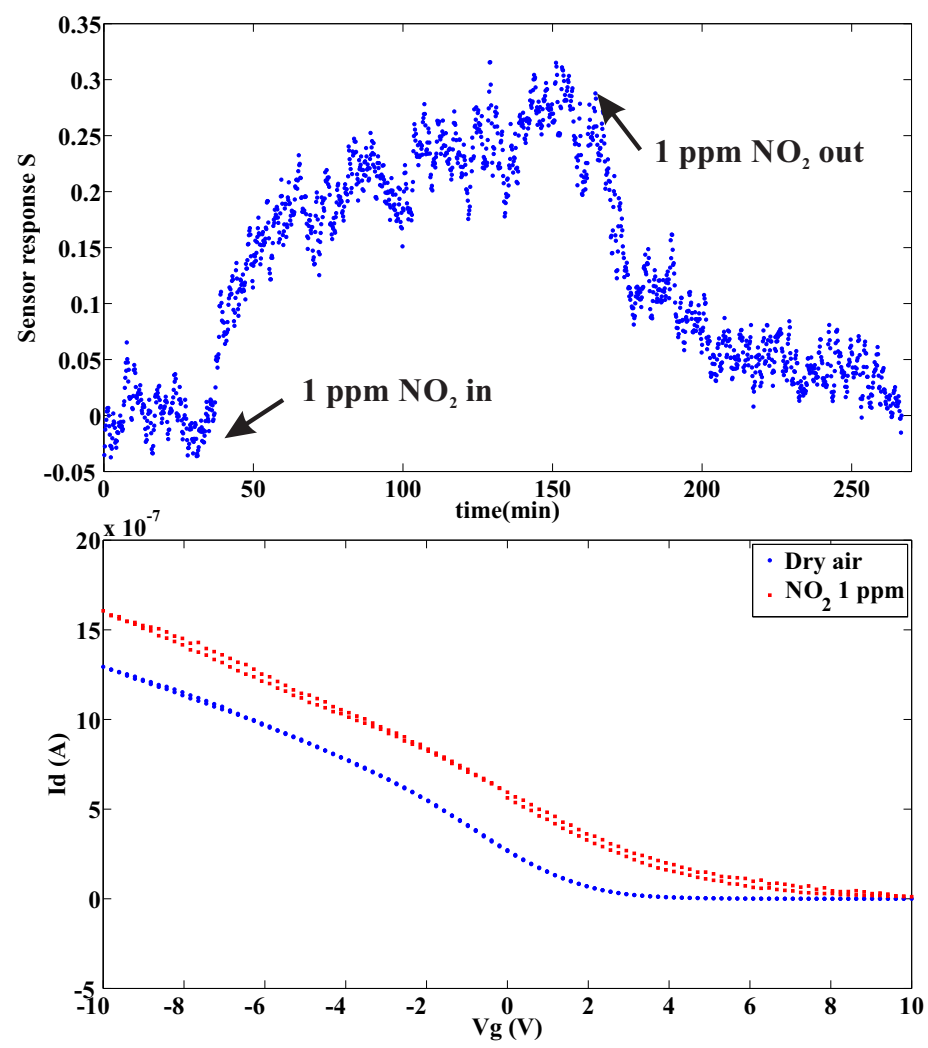

Fig. 3. The sensor response of a typical suspended carbon nanotube gas sensor.

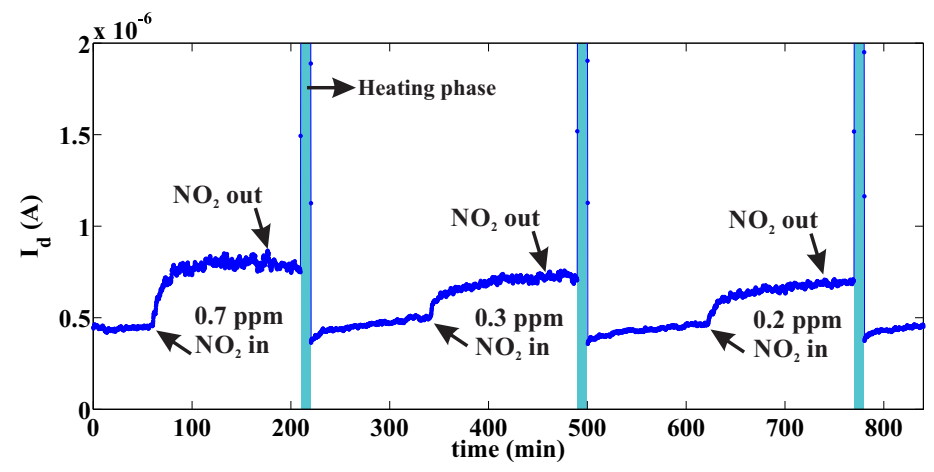

Fig. 4. Sensor recovery by self-heating where the carrier gas is dry air. After exposure to various concentrations of $\mathrm{NO}_{2}$, the sensor recovers through self-heating which is induced by an increased bias on the sensor [14].

MOX sensor heater. The power consumption of that surrounding circuitry overcomes the power consumption of the carbon nanotube. With ultra-low-power components such as [27], the conditioning circuit would consume about $20 \mu \mathrm{W}$.

\section{Simulation SetuP}

To evaluate the proposed approach in terms of power consumption reduction and lifetime extension, simulations in Matlab have been implemented using the power consumption measurements of the nanotube and gas sensor device described in the previous section. We assume that the sensor node is powered with two batteries providing $3000 \mathrm{mAh}$ capacity. Three event-driven wake-up operating modes have been modeled:
W1) The MOX gas sensor is activated only by the carbon nanotube sensor interrupt. In this case, there is no active wireless communication between the nodes and the radio is kept off during the node operation. We consider three cases: when the event (i.e., the interrupt generated by the nanotube upon gas detection) occurs twice a day, once a day, and once in 2 days. As the carbon nanotube requires 10 minutes to recover, it cannot detect more than 6 alarms per hour. In the recovery period the MOX gas sensor works in duty cycling D. Thus, critical gas concentrations are detected by the MOX if they occur during the nanotube recovery period.

W2) The MOX gas sensor is activated by either the trigger from the carbon nanotube sensor or by the trigger from the neighbour node in the wireless sensor network via wireless radio. In this scenario the node has a radio with a dutycycled activity which increases the energy consumption, but it also increases the promptness of detecting dangerous gas concentrations (especially during the critical time where the carbon nanotube is in recovery process and MOX is dutycycled). We explore the situations with alarms that occur twice a day, once a day, and once every two days. We set the radio duty cycle to $0.1 \%$. Supposing that the radio needs maximum $2 \mathrm{~ms}$ to receive a message, this duty cycle value introduces a maximal delay of $2 \mathrm{~s}$.

W3) The MOX gas sensor is activated by the carbon nanotube sensor interrupt and it is also heated periodically, with a very low duty cycle $(0.1 \%)$. In that way, we increase the probability of detecting critical events.

To have the comparison with a traditional MOX gas sensor node and to evaluate the benefits of the nanotube, we model three duty-cycled operating modes. In the following modes, we suppose the sensor node does not include a carbon nanotube as a low-power gas detector, but it detects the gas concentration increment only with the MOX gas sensor, as follows:

D1) The MOX gas sensor operating with a fixed duty cycle $D(1 \%, 2 \%, 5 \%$, and $10 \%$, respectively). The gas sensor is periodically heated for 2 seconds (until the steady-state is reached) and then the heater switches off. The longer the heater is off, the lower is the energy consumption, but the delay in response and the risk of missing an event is longer. For example, when $D=1 \%$, the inactive time it is 198 seconds.

D2) The MOX gas sensor operating with an adaptive duty cycle $D$ (during the day it switches between $1 \%, 2 \%, 5 \%$, and $10 \%$ duty cycles according to some predefined rules and the information about the gas concentration obtained from the neighbour nodes). For $2 \mathrm{~h}$ in a day we have $10 \%$ duty cycle, for $10 \mathrm{~h}$ in a day $5 \%$ duty cycle, for another $10 \mathrm{~h}$ in a day $2 \%$ duty cycle and for $2 \mathrm{~h}$ in a day $1 \%$ duty cycle. Likewise W2 scenario, this scenario is also appropriate for wireless sensor networks. In this case the radio duty cycles with $0.1 \%$.

D3) The MOX gas sensor continuously heated. Here the response to the concentration increment is instant, but the energy consumption is very high and thus not suitable for a battery-operated device. However, this comparison is useful to highlight the energy issue of MOX sensors and the benefits of power management. 


\section{Simulation RESUlTS}

Fig. 5 shows the simulated lifetime of the presented sensor node in the above mentioned different operating modes (W1W3 and D1-D3). We can notice that the nanotube approaches have longer lifetimes than all the duty-cycling approaches. Comparing the lifetime of the scenario where the MOX sensor is woken up only from the carbon nanotube (W1) to the scenario where the wake up can come from both nanotube and neighbor node (W2), we notice about $11 \%$ lifetime decrement caused by the radio overhead. When we compare the lifetime of the sensor wake-up with the adaptive duty cycle (D2) to the nanotube mode with the longest lifetime (W1), we notice $35 \%$ of lifetime improvement. When the radio is also included (W2), the increment is $21 \%$.

An important issue with the current nanotube technology is the recovery time. The results in [14] indicate a recovery time of 10 minutes, which was used in our calculations. Fig. 6 shows worst-case reaction time for every operating mode where this effect is highlighted. For the scenario W1, the reaction time is limited with the nanotube response and its recovery time (10 minutes). The same worst-case delay is for the scenario W3 where, except the nanotube, we have a MOX gas sensor in duty-cycled mode. However, the used duty cycle is rather low, which introduces a larger delay than the nanotube recovery time. On the other hand, in mode W2, the worst-case reaction time is defined by the radio reaction time. For the used duty cycle of $0.1 \%$, maximal delay is 4 seconds ( 2 seconds for the inactive radio time and 2 seconds for MOX sensor activation time). For the scenarios D1, the maximal reaction time is defined by the duty cycle (it is from 20 to 200 seconds). In adaptive MOX duty-cycling mode with radio duty cycle (D2), the delay is again determined by the radio (i.e., $4 \mathrm{~s}$ delay $-2 \mathrm{~s}$ for the radio and $2 \mathrm{~s}$ for the MOX sensor). For the continuous mode, the delay is only 2 seconds (the time necessary for the MOX sensor to determine gas concentration after activation).

These simulation results show that the nanotube gas sensor is beneficial for increasing the lifetime of the MOX sensor based air quality monitoring system, but increases the reaction time due to its long recovery. That can be overcome by introducing the radio communication in the case of increased gas concentration, where the radio of the sensor node has a low duty cycle (to lower the energy consumption overhead but still enhance the promptness). Further investigations can also focus on reducing the recovery time of the nanotube sensor since this will make the carbon nanotube a perfect gas detector to be coupled with traditional MOX to increase the energy efficiency. By increasing the temperature of the nanotube during the self-heating phase, it may be possible to accelerate the desorption even further in accordance with the Arrhenius law. However, the maximum heating temperature is limited by the onset of decomposition of the nanotube, which can occur at about $600^{\circ} \mathrm{C}$.

Another issue with the present design is that the carbon nanotube requires conditioning circuitry that consumes much more power than the carbon nanotube itself and partially reduces the benefits of the ultra low power consumption $(40 \mathrm{nW})$. Furthermore, the cross-sensitivity of nanotubes to other gases may create false alarms which needs to be addressed in future work. On the other hand, studies show that nanotube surface functionalization could render them sensitive to other

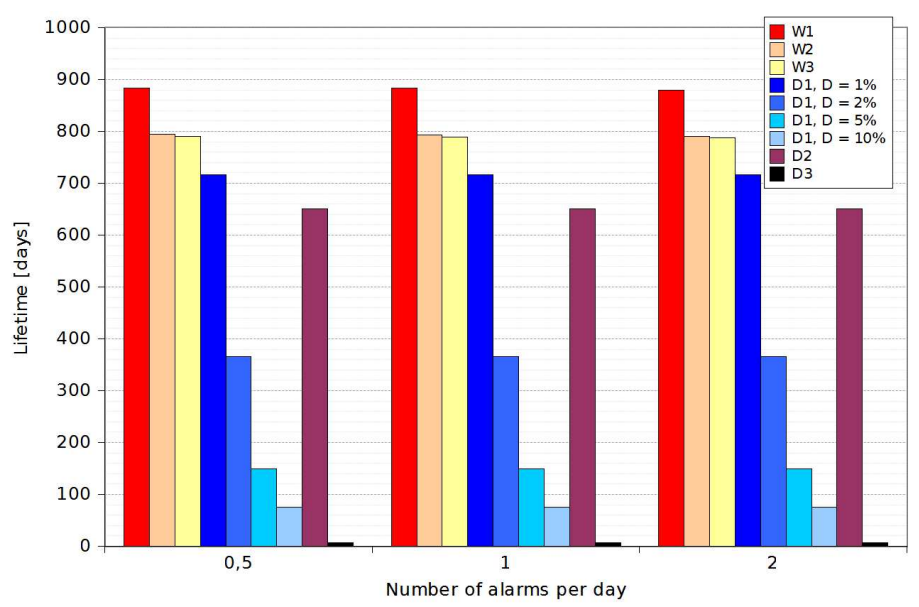

Fig. 5. Predicted lifetime of the proposed node, for different operating modes.

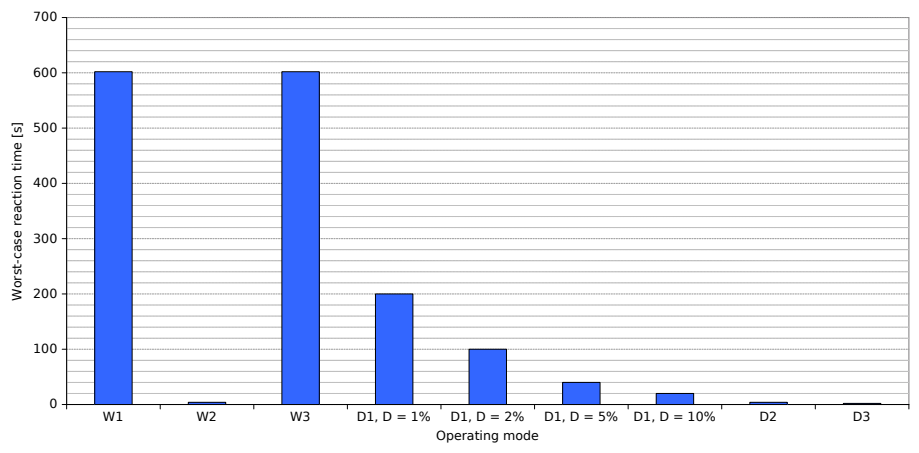

Fig. 6. Minimal worst-case reaction time for different operating modes.

gases [22], which may enable their application as a low-power trigger for other gases as well.

\section{CONCLUSION}

In this paper, we propose a novel concept of sensor wakeup for energy-efficient smart gas monitoring. Our system benefits from the low-power activity (enabled by the carbon nanotube [14]) and acceptable response time (enabled by the MOX gas sensor). Simulations of realistic power consumption values demonstrate carbon nanotube gas sensors, despite their technological immaturity, already enable a significant lifetime prolongation of the node, of up to $20 \%-35 \%$, depending on the scenario.

As a future work, we will explore possibility of adding another carbon nanotube, that can be used as a back-up sensor while the main nanotube is in recovery state. Moreover, we will study the possibilities of incorporating a wake-up receiver $[28,29]$ in the node design, which will allow receiving alarm messages from neighbour nodes without the need for duty-cycling. Another important issue is to ensure the stability of the MOX gas sensor response after a longer inactive period.

\section{ACKNOWLEDGMENT}

This work has been partly supported by the European Regional Development Fund under the project "Advanced technologies in power plants and rail vehicles", by the EU H2020 
project Flexmeter (no. 646568), by the SNF projects "Transient Computing Systems" (no. 200021 157048) and "Enhancing research and development of energy-efficient networked sensor systems in South Eastern Europe" (no. IZ74Z0 160481), and by the ETH Zurich Grant funding.

\section{REFERENCES}

[1] A. Kerhet, F. Leonardi, A. Boni, P. Lombardo, M. Magno, and L. Benini, "Distributed video surveillance using hardware-friendly sparse large margin classifiers," in IEEE Conference on Advanced Video and Signal Based Surveillance (AVSS). IEEE, 2007, pp. 87-92.

[2] M. Magno, D. Brunelli, L. Thiele, and L. Benini, "Adaptive power control for solar harvesting multimodal wireless smart camera," in Third ACM/IEEE International Conference on Distributed Smart Cameras (ICDSC). IEEE, 2009, pp. 1-7.

[3] A. E. Şuşu, M. Magno, A. Acquaviva, D. Atienza, and G. De Micheli, "Reconfiguration strategies for environmentally powered devices: theoretical analysis and experimental validation," in Transactions on HighPerformance Embedded Architectures and Compilers I. Springer, 2007, pp. $341-360$.

[4] M. Magno, T. Polonelli, L. Benini, and E. Popovici, "A low-cost, highly scalable wireless sensor network solution to achieve smart led light control for green buildings," IEEE Sensors J., vol. 15, no. 5, pp. 29632973, Apr. 2015.

[5] Air Quality Standards, European Commission Std. [Online]. Available: http://ec.europa.eu/environment/air/quality/standards.htm

[6] National Ambient Air Quality Standards, Unated States Environmental Protection Agency Std. [Online]. Available: http://www.epa.gov/air/criteria.html

[7] N. Nikzad, N. Verma, C. Ziftci, E. Bales, N. Quick, P. Zappi, K. Patrick, S. Dasgupta, I. Krueger, T. S. Rosing, and W. G. Griswold, "Citisense: Improving geospatial environmental assessment of air quality using a wireless personal exposure monitoring system," in Proceedings Wireless Health '12, San Diego, CA, USA, Oct. 23-25 2012.

[8] S. S. David Hasenfratz, Olga Saukh and L. Thiele, "Participatory air pollution monitoring using smartphones," in Proceedings 2nd International Workshop on Mobile Sensing, Beijing, China, Apr. 16-20 2012 pp. 1-5.

[9] D. Oletic and V. Bilas, "Design of sensor node for air quality crowdsensing," in Proceedings of IEEE Sensors Applications Symposium (SAS), Zadar, Croatia, Apr. 13-15 2015, pp. 346-350.

[10] V. Jelicic, M. Magno, D. Brunelli, G. Paci, and L. Benini, "Contextadaptive multimodal wireless sensor network for energy-efficient gas monitoring," IEEE Sensors J., vol. 13, no. 1, pp. 328-338, Jan 2013.

[11] X. Liu, S. Cheng, H. Liu, S. Hu, D. Zhang, and H. Ning, "A survey on gas sensing technology," Sensors, vol. 12, no. 7, pp. 9635-9665, 2012.

[12] D. Oletic and V. Bilas, "Empowering smartphone users with sensor node for air quality measurement," Journal of Physics: Conference Series, vol. 450, no. 1, p. 012028, 2013.

[13] A. Boyd, I. Dube, G. Fedorov, M. Paranjape, and P. Barbara, "Gas sensing mechanism of carbon nanotubes: From single tubes to high-density networks," Carbon, vol. 69, no. $0, \quad$ pp. $417-423,2014$. [Online]. Available: http://www.sciencedirect.com/science/article/pii/S0008622313011998

[14] K. Chikkadi, M. Muoth, V. Maiwald, C. Roman, and C. Hierold, "Ultralow power operation of self-heated, suspended carbon nanotube gas sensors," Applied Physics Letters, vol. 103, no. 223109, 2013.
[15] P. Clement, A. Ramos, A. Lazaro, L. Molina-Luna, C. Bittencourt, D. Girbau, and E. Llobet, "Oxygen plasma treated carbon nanotubes for the wireless monitoring of nitrogen dioxide levels," Sensors and Actuators B: Chemical, vol. 208, no. 0, pp. 444 - 449, 2015. [Online]. Available: http://www.sciencedirect.com/science/article/pii/S0925400514014385

[16] I. Sayhan, A. Helwig, T. Becker, G. Muller, I. Elmi, S. Zampolli, M. Padilla, and S. Marco, "Discontinuously operated metal oxide gas sensors for flexible tag microlab applications," IEEE Sensors J., vol. 8, no. 2, pp. 176-181, 2008.

[17] D. Oletic, V. Jelicic, D. Antolovic, and V. Bilas, "Energy-efficient atmospheric CO concentration sensing with on-demand operating MOX gas sensor," in IEEE Sensors Conf., Valencia, Spain, Nov. 2-5 2014, pp. 795-798.

[18] V. Jelicic, M. Magno, G. Paci, D. Brunelli, and L. Benini, "Design, characterization and management of a wireless sensor network for smart gas monitoring," in 4th IEEE International Workshop on Advances in Sensors and Interfaces (IWASI), Savelletri di Fasano, Italy, 29-29 June 2011, pp. 115-120.

[19] S. Bicelli, A. Depari, G. Faglia, A. Flammini, A. Fort, M. Mugnaini, A. Ponzoni, V. Vignoli, and S. Rocchi, "Model and experimental characterization of the dynamic behavior of low-power carbon monoxide MOX sensors operated with pulsed temperature profiles," IEEE Trans. on Instrumentation and Measurement, vol. 58, no. 5, pp. 1324-1332, 2009.

[20] M. Rossi and D. Brunelli, "Ultra low power MOX sensor reading for natural gas wireless monitoring," IEEE Sensors J., vol. 14, no. 10, pp. 3433-3441, Oct. 2014.

[21] V. Jelicic, D. Oletic, T. Sever, and V. Bilas, "Evaluation of mox gas sensor transient response for low-power operation," in Proceedings of IEEE Sensors Applications Symposium (SAS), Zadar, Croatia, Apr. 1315 2015, pp. 108-112.

[22] Y. L. A. Hannon and, J. Li, and M. Meyyappan, "Room temperature carbon nanotube based sensor for carbon monoxide detection," Journal of Sensors and Sensor Systems, vol. 3, pp. 349-354, Dec. 2014.

[23] H. Guerin, H. L. Poche, R. Pohle, E. Buitrago, M. F.-B. Badia, J. Dijon, and A. M. Ionescu, "Carbon nanotube gas sensor array for multiplex analyte discrimination," Sensors and Actuators B: Chemical, vol. 207, Part A, no. 0, pp. 833 - 842, 2015. [Online]. Available: http://www.sciencedirect.com/science/article/pii/S092540051401329X

[24] K. Chikkadi, M. Muoth, N. Beckmann, C. Roman, and C. Hierold, "Supression of cross-sensitivity to humidity in pristine, suspended singlewalled nanotube NO2 sensors," in XXVIII Eurosensors, 2014, pp. 704 707.

[25] "Wispes W24TH." [Online]. Available: http://www.wispes.com/products-page/wireless-sensors/wispes-w24th/

[26] MiCS-2710 NO2 Sensor. [Online]. Available: http://www.cdiweb.com/datasheets/e2v/mics-5525.pdf

[27] TPS61222 - Tiny Low Input Voltage Boost Converter, Texas Instruments, 2014. [Online]. Available: http://www.ti.com/product/tps61222

[28] M. Magno and L. Benini, "An ultra low power high sensitivity wake-up radio receiver with addressing capability," in Proc. IEEE 10th International Conference on Wireless and Mobile Computing, Networking and Communications (WiMob), Larnaca, Cyprus, Oct. 8-10 2014, pp. 92-99.

[29] V. Jelicic, M. Magno, D. Brunelli, V. Bilas, and L. Benini, "Benefits of wake-up radio in energy-efficient multimodal surveillance wireless sensor network," IEEE Sensos J., vol. 14, no. 9, pp. 3210-3220, Sept. 2014 . 